\title{
Strongly convex set-valued maps
}

\author{
Hugo Leiva • Nelson Merentes • Kazimierz Nikodem • \\ José Luis Sánchez
}

Received: 2 August 2012 / Accepted: 19 February 2013 / Published online: 12 March 2013

(C) The Author(s) 2013. This article is published with open access at Springerlink.com

\begin{abstract}
We introduce the notion of strongly $t$-convex set-valued maps and present some properties of it. In particular, a Bernstein-Doetsch and Sierpiński-type theorems for strongly midconvex set-valued maps, as well as a Kuhn-type result are obtained. A representation of strongly $t$-convex set-valued maps in inner product spaces and a characterization of inner product spaces involving this representation is given. Finally, a connection between strongly convex set-valued maps and strongly convex sets is presented.
\end{abstract}

Keywords Strongly convex function - Strongly convex set-valued map ·

Strongly convex set

Mathematics Subject Classification (2010) Primary 26B25 - Secondary 54C60, 46C15, 39B62

\section{Introduction}

Let $(X,\|\cdot\|)$ be a normed space, $D$ be a convex subset of $X$ and let $c>0$. A function $f: D \rightarrow \mathbb{R}$ is called strongly convex with modulus $c$ if

H. Leiva

Departamento de Matemáticas, Facultad de Ciencias, Universidad de los Andes, Mérida, Venezuela e-mail: hleiva@ula.ve

N. Merentes · J. L. Sánchez

Escuela de Matemáticas, Universidad Central de Venezuela, Caracas, Venezuela

N. Merentes

e-mail: nmerucv@gmail.com

J. L. Sánchez

e-mail: jose.sanchez@ciens.ucv.ve

K. Nikodem $(\bowtie)$

Department of Mathematics and Computer Science, University of Bielsko-Biała, ul. Willowa 2,

43-309 Bielsko-Biała, Poland

e-mail: knikodem@ath.bielsko.pl 


$$
f\left(t x_{1}+(1-t) x_{2}\right) \leq t f\left(x_{1}\right)+(1-t) f\left(x_{2}\right)-c t(1-t)\left\|x_{1}-x_{2}\right\|^{2}
$$

for all $x_{1}, x_{2} \in D$ and $t \in[0,1]$. Strongly convex functions have been introduced by Polyak [27]. He used them for proving the convergence of a gradient type algorithm for minimizing a function. They have many properties useful in optimization theory and mathematical economics. For instance, if $f$ is a strongly convex function then its level sets $\{x: f(x) \leq \lambda\}$ are bounded for each $\lambda$ and $f$ has a unique minimum on every closed convex set. Numerous properties and applications of them can be found in the literature (see, for instance, $[9,13,14,17,19,20,26,29,35-37])$. Recently, Huang [11,12] extended the definition (1) of strongly convex functions to set-valued maps. He used such maps to investigate error bounds for some inclusion problems with set constraints.

In this note we introduce the notion of strongly $t$-convex set-valued maps (strongly midconvex, in particular) and present some properties of it. Our definition is weaker than that given by Huang, but is easier to verify than that one since we assume that relation (2) below holds for only one fixed $t \in(0,1)$, not for all $t \in(0,1)$. Therefore, it seems to be interesting and important that under weak regularity assumptions the class of strongly t-convex set-valued maps coincides with the class of strongly convex set-valued maps. We present, in Sect. 3, some counterparts of the classical theorems of Bernstein-Doetsch and Sierpiński for strongly midconvex set-valued maps. We give also a version of Huang's theorem on error bounds for some inclusion optimization problems as an application of these results. In Sect. 4 we prove a Kuhn-type theorem stating that strongly $t$-convex set-valued maps are strongly midconvex. Section 5 contains some representation of strongly $t$-convex set valued maps with domain in an inner product space and a characterization of inner product spaces involving this representation. Finally, in Sect. 6, we discuss connections of strongly convex set-valued maps with strongly convex sets.

\section{Preliminaries}

Let $(X,\|\cdot\|)$ and $(Y,\|\cdot\|)$ be real normed spaces and $D$ be a convex subset of $X$. Throughout this paper $B$ denotes the open unit ball in $Y$ and $\bar{B}$ the closure of $B$. We denote by $n(Y)$ the family of all nonempty subsets of $Y$, and by $b(Y), \operatorname{cl}(Y), b c l(Y)$ and $\operatorname{ccl}(Y)$ the subfamilies of $n(Y)$ of all bounded, closed, bounded closed and convex closed sets, respectively.

Let $t \in(0,1)$ and $c>0$. We say that a set-valued map $F: D \rightarrow n(Y)$ is stronglyt-convex with modulus $c$ if

$$
t F\left(x_{1}\right)+(1-t) F\left(x_{2}\right)+c t(1-t)\left\|x_{1}-x_{2}\right\|^{2} \bar{B} \subset F\left(t x_{1}+(1-t) x_{2}\right),
$$

for all $x_{1}, x_{2} \in D$. We say that $F$ is strongly midconvex with modulus $c$ if it satisfies (2) with $t=1 / 2$, that is

$$
\frac{1}{2} F\left(x_{1}\right)+\frac{1}{2} F\left(x_{2}\right)+\frac{c}{4}\left\|x_{1}-x_{2}\right\|^{2} \bar{B} \subset F\left(\frac{x_{1}+x_{2}}{2}\right),
$$

for all $x_{1}, x_{2} \in D$. F is called strongly convex with modulus $c$ if it satisfies (2) with every $t \in(0,1)$ and all $x_{1}, x_{2} \in D$ (see $\left.[11,12]\right)$. The usual notions of $t$-convex, midconvex and convex set-valued maps correspond to relations (2) and (3) with $c=0$, respectively (cf. e.g. $[3-7,21,34])$.

Clearly, the definition of strongly convex (strongly $t$-convex, strongly midconvex) setvalued maps is motivated by the definition of strongly convex functions. In the following 
example we show some connections between conditions (1) and (2) in the case where $F$ has values in $\mathbb{R}$.

Example 1 Let $D$ be a convex subset of $X$. Assume that $f: D \rightarrow \mathbb{R}$ is strongly convex with modulus $c, g: D \rightarrow \mathbb{R}$ is strongly concave with modulus $c$ (that is $-g$ satisfies (1)) and $f \leq g$ on $D$. Then, one can easily check that the set-valued maps $F_{1}, F_{2}, F_{3}$ defined by

$$
F_{1}(x)=[f(x), g(x)], \quad F_{2}(x)=[f(x), \infty), \quad F_{3}(x)=(-\infty, g(x)], x \in D,
$$

are strongly convex with modulus $c$. In particular, the set-valued map $F:[-r, r] \rightarrow b c l(\mathbb{R})$ given by $F(x)=c\left[x^{2}-r^{2}, r^{2}-x^{2}\right], x \in[-r, r]$, is strongly convex with modulus $c$ (because $f(x)=c\left(x^{2}-r^{2}\right)$ is strongly convex with modulus $\left.c\right)$. Conversely, if $F: D \rightarrow b(\mathbb{R})$ is strongly convex with modulus $c$, then the function $f: D \rightarrow \mathbb{R}$ given by $f(x)=\inf F(x)$, $x \in D$, is strongly convex with modulus $c$ and the function $g(x)=\sup F(x), x \in D$, is strongly concave with modulus $c$.

The following lemmas will be used in the sequel.

Lemma 1 If $F: D \rightarrow n(Y)$ is strongly midconvex with modulus $c$ then

$$
\frac{k}{2^{n}} F\left(x_{1}\right)+\left(1-\frac{k}{2^{n}}\right) F\left(x_{2}\right)+c \frac{k}{2^{n}}\left(1-\frac{k}{2^{n}}\right)\left\|x_{1}-x_{2}\right\|^{2} \bar{B} \subset F\left(\frac{k}{2^{n}} x_{1}+\left(1-\frac{k}{2^{n}}\right) x_{2}\right),
$$

for all $x_{1}, x_{2} \in D$ and all $k, n \in \mathbb{N}$ such that $k<2^{n}$.

Proof The proof is by induction on $n$. For $n=1$ (4) reduces to (3). Assuming (4) to hold for some $n \in \mathbb{N}$ and all $k<2^{n}$, we will prove it for $n+1$. Fix $x_{1}, x_{2} \in D$ and take $k<2^{n+1}$. Without loss of generality it suffices to consider only the case $k<2^{n}$ equivalent to $\frac{k}{2^{n+1}}<\frac{1}{2}$ (indeed, for $k=2^{n}$ we get (3), whereas the case $2^{n}<k<2^{n+1}$ is symmetric to the previous one because then $\left(1-\frac{k}{2^{n+1}}\right)<\frac{1}{2}$ ). Using the induction assumption and the definition (3) of strong midconvexity, we get

$$
\begin{aligned}
& \frac{k}{2^{n+1}} F\left(x_{1}\right)+\left(1-\frac{k}{2^{n+1}}\right) F\left(x_{2}\right)+c \frac{k}{2^{n+1}}\left(1-\frac{k}{2^{n+1}}\right)\left\|x_{1}-x_{2}\right\|^{2} \bar{B} \\
& \subset \frac{1}{2}\left[\frac{k}{2^{n}} F\left(x_{1}\right)+\left(1-\frac{k}{2^{n}}\right) F\left(x_{2}\right)+c \frac{k}{2^{n}}\left(1-\frac{k}{2^{n}}\right)\left\|x_{1}-x_{2}\right\|^{2} \bar{B}\right]+\frac{1}{2} F\left(x_{2}\right) \\
& \quad+\frac{c k^{2}}{4\left(2^{n}\right)^{2}}\left\|x_{1}-x_{2}\right\|^{2} \bar{B} \\
& \subset \frac{1}{2} F\left(\frac{k}{2^{n}} x_{1}+\left(1-\frac{k}{2^{n}}\right) x_{2}\right)+\frac{1}{2} F\left(x_{2}\right)+\frac{c}{4}\left\|\left(\frac{k}{2^{n}} x_{1}+\left(1-\frac{k}{2^{n}}\right) x_{2}\right)-x_{2}\right\|^{2} \bar{B} \\
& \subset F\left(\frac{k}{2^{n+1}} x_{1}+\left(1-\frac{k}{2^{n+1}}\right) x_{2}\right),
\end{aligned}
$$

which finishes the proof.

Lemma 2 Let $t \in(0,1)$. If $F: D \rightarrow c l(Y)$ is $t$-convex (strongly $t$-convex with modulus $c$ in particular), then $F(x)$ is convex for every $x \in D$.

Proof For every $x \in D, t F(x)+(1-t) F(x) \subset F(x)$ by (2). Since $F(x)$ is closed, it follows that it is convex (see e.g. [1]).

Recall also the Rådström cancelation law [28] which is a useful tool in our investigation.

Lemma 3 Let $A, B, C$ be subsets of $X$ such that $A+C \subset B+C$. If $B$ is closed convex and $C$ is bounded and nonempty, then $A \subset B$. 


\section{Bernstein-Doetsch-type results}

Obviously, every strongly convex set-valued map is strongly $t$-convex with any $t \in(0,1)$, but the converse is not true, in general. For instance, if $a: \mathbb{R} \rightarrow \mathbb{R}$ is an additive discontinuous function [such functions can be constructed by use of the Hamel basis (cf. e.g. [15,31])], then the set-valued map $F:[-1,1] \rightarrow \operatorname{bcl}(\mathbb{R})$ defined by $F(x)=\left[x^{2}-1,1-x^{2}\right]+$ $a(x)$, is strongly midconvex with modulus 1 , but is not strongly convex (with any modulus). Indeed, the function $f:[-1,1] \rightarrow \mathbb{R}$ given by $f(x)=\inf F(x)=x^{2}-1+a(x)$ is strongly midconvex with modulus 1 , but is not strongly convex because of the discontinuity (cf. Example 1). However, in the class of continuous set-valued maps, strong midconvexity ( $t$-convexity) is equivalent to strong convexity. In fact, this equivalence holds under conditions much weaker than continuity. In this section we present a few results of such type.

A set-valued function $F: D \rightarrow n(Y)$ is said to be continuous (with respect to the Hausdorff topology on $n(Y))$ at a point $x_{0} \in D$ if for every $\varepsilon>0$ there exists a $\delta>0$ such that

$$
F\left(x_{0}\right) \subset F(x)+\varepsilon B
$$

and

$$
F(x) \subset F\left(x_{0}\right)+\varepsilon B
$$

for every $x \in D$ such that $\left\|x-x_{0}\right\|<\delta$. If we assume only condition (5) (condition (6)) $F$ is said to be lower semicontinuous (upper semicontinuous) at $x_{0}$.

Theorem 4 If a set-valued function $F: D \rightarrow b c l(Y)$ is strongly midconvex with modulus $c$ and upper semicontinuous on $D$, then it is strongly convex with modulus $c$.

Proof Let $x_{1}, x_{2} \in D$ and $t \in(0,1)$. Take a sequence $\left(q_{n}\right)$ of dyadic numbers in $(0,1)$ tending to $t$ and fix an $\varepsilon>0$. Since the set-valued functions of the form $\mathbb{R} \ni s \rightarrow s A \in n(Y)$ are continuous provided the set $A$ is bounded (see e.g. [22], Lemma 3.2), we have

$$
t F\left(x_{1}\right) \subset q_{n} F\left(x_{1}\right)+\frac{\varepsilon}{4} \bar{B}, \quad(1-t) F\left(x_{2}\right) \subset\left(1-q_{n}\right) F\left(x_{2}\right)+\frac{\varepsilon}{4} \bar{B}
$$

and

$$
c t(1-t)\left\|x_{1}-x_{2}\right\|^{2} \bar{B} \subset c q_{n}\left(1-q_{n}\right)\left\|x_{1}-x_{2}\right\|^{2} \bar{B}+\frac{\varepsilon}{4} \bar{B},
$$

for all $n \geq n_{1}$. On the other hand, by the upper semicontinuity of $F$ at the point $t x_{1}+(1-t) x_{2}$, we get

$$
F\left(q_{n} x_{1}+\left(1-q_{n}\right) x_{2}\right) \subset F\left(t x_{1}+(1-t) x_{2}\right)+\frac{\varepsilon}{4} \bar{B},
$$

for all $n \geq n_{2}$. Hence, using (7), (8), (9) and Lemma 1, we obtain

$$
\begin{aligned}
& t F\left(x_{1}\right)+(1-t) F\left(x_{2}\right)+c t(1-t)\left\|x_{1}-x_{2}\right\|^{2} \bar{B} \\
& \quad \subset q_{n} F\left(x_{1}\right)+\left(1-q_{n}\right) F\left(x_{2}\right)+c q_{n}\left(1-q_{n}\right)\left\|x_{1}-x_{2}\right\|^{2} \bar{B}+\frac{3}{4} \varepsilon \bar{B} \\
& \quad \subset F\left(q_{n} x_{1}+\left(1-q_{n}\right) x_{2}\right)+\frac{3}{4} \varepsilon \bar{B} \subset F\left(t x_{1}+(1-t) x_{2}\right)+\varepsilon \bar{B},
\end{aligned}
$$

for all $n \geq \max \left\{n_{1}, n_{2}\right\}$. Since the above inclusions hold for every $\varepsilon>0$, we have also

$$
\begin{aligned}
& t F\left(x_{1}\right)+(1-t) F\left(x_{2}\right)+c t(1-t)\left\|x_{1}-x_{2}\right\|^{2} \bar{B} \\
& \quad \subset \bigcap_{\varepsilon>0}\left(F\left(t x_{1}+(1-t) x_{2}\right)+\varepsilon \bar{B}\right)=\operatorname{cl} F\left(t x_{1}+(1-t) x_{2}\right)=F\left(t x_{1}+(1-t) x_{2}\right) .
\end{aligned}
$$


This shows that $F$ is strongly convex with modulus $c$ and finishes the proof.

It is known that if a midconvex set-valued map $F: D \rightarrow b(Y)$, defined on an open convex set $D \subset X$, satisfies some regularity assumptions such as lower semicontinuity at a point of $D$ or boundedness on a set with nonempty interior or measurability, then it is continuous (see, for instance $[22,23,34]$ and the references therein). Therefore, as a consequence of Theorem 4 and those results we obtain the following corollaries. Here $D$ is assumed to be an open convex subset of $X$.

Corollary 5 If a set-valued map $F: D \rightarrow b c l(Y)$ is strongly midconvex with modulus $c$ and lower semicontinuous at a point of $D$, then it is continuous and strongly convex with modulus $c$.

Now, let us adopt the following definition which is a natural extension of the concept of the boundedness for real-valued functions. A set-valued map $F: D \rightarrow n(Y)$ is said to be bounded on a set of $A \subset D$, if there exists a number $r>0$ such that $F(x) \cap r \bar{B} \neq \varnothing$ for every $x \in A$. The following result is an analogue of the classical theorem of Bernstein and Doetsch (see $[15,31])$.

Corollary 6 If a set-valued map $F: D \rightarrow b c l(Y)$ is strongly midconvex with modulus $c$ and bounded on a subset of $D$ with a nonempty interior, then it is continuous and strongly convex with modulus $c$.

Recall that a set-valued map $F: \mathbb{R}^{n} \supset D \rightarrow n(Y)$ is Lebesgue measurable if for every open set $W \subset Y$ the set $F^{+}(W)=\{x \in D: F(x) \subset W\}$ is Lebesgue measurable. The next result is a counterpart of the theorem of Sierpiński (see [15,31]).

Corollary 7 If a set-valued map $F: \mathbb{R}^{n} \supset D \rightarrow \operatorname{bcl}(Y)$ is strongly midconvex with modulus $c$ and Lebesgue measurable, then it is continuous and strongly convex with modulus $c$.

Remark 8 Convex set-valued maps arise naturally from, e.g., the constraints of convex optimization problems and play an important role in various questions of convex analysis and economic theory (see, for instance, $[4,5,32,33]$ ). Sometimes we can replace the assumption that the set-valued map under consideration is (strongly) convex by the weaker and easier to verify one-that it is (strongly) midconvex, and, consequently, we get formally stronger results. As an example we present a version of Huang's theorem (cf. [11], Theorem 4.5) on error bounds for some inclusion optimization problem (see [11] for further details). Using Corollaries 5-7 above and the fact that continuous closed-valued maps have closed graphs (see e.g. [30,33]), we get the following variant of Huang's theorem (in which instead of the assumptions that $F$ is strongly convex with closed graph we assume that it is strongly midconvex and lower semicontinuous at a point).

Theorem 9 Let $X, Y$ be Banach spaces, $D$ be a convex open subset of $X$ and $F: D \rightarrow b c l(Y)$ be strongly midconvex with modulus $c$ and lower semicontinuous at a point of $D$ (or bounded on a subset of $D$ with nonempty interior or Lebesgue measurable (in the case where $\left.X=\mathbb{R}^{n}\right)$ ). Let $b \in F(D)$ and $\eta>0$. If for each $a \in b d\left(F^{-1}(b)\right)$ there exists $\delta_{a}>0$ such that

$$
\eta\left[d\left(x, F^{-1}(b)\right)\right]^{2} \leq d(b, F(x))
$$

for all $x \in a+\delta_{a} B$, then there exists $\tau:=\min \{c, \eta\}$ such that

$$
\tau\left[d\left(x, F^{-1}(b)\right)\right]^{2} \leq d(b, F(x))
$$

for all $x \in D$. 


\section{Kuhn-type result}

It is known by the Kuhn's theorem [16] that $t$-convex functions (with arbitrarily fixed $t \in(0,1))$ are midconvex. Similar results hold also for $t$-convex set-valued maps [7] and for strongly $t$-convex functions [2]. In this section we present a counterpart of those results for strongly $t$-convex set-valued maps. In the proof we apply the idea used in [8].

Theorem 10 Let $(X,\|\cdot\|)$ and $(Y,\|\cdot\|)$ be real normed spaces, $D$ be a convex subset of $X$ and $t \in(0,1)$ be a fixed number. If a set-valued function $F: D \rightarrow \operatorname{bcl}(Y)$ is strongly $t$-convex with modulus $c$, then it is strongly midconvex with modulus $c$.

Proof Fix $x_{1}, x_{2} \in D$ and put $z:=\frac{x_{1}+x_{2}}{2}, u:=(1-t) x_{1}+t z$ and $v:=(1-t) z+t x_{2}$. Then

$$
z=t u+(1-t) v
$$

Since

$$
\left\|x_{1}-z\right\|=\left\|z-x_{2}\right\|=\|u-v\|=\frac{1}{2}\left\|x_{1}-x_{2}\right\|
$$

we have

$$
\begin{aligned}
\frac{1}{2} t(1-t)\left\|x_{1}-x_{2}\right\|^{2} \bar{B}= & t^{2}(1-t)\left\|x_{1}-z\right\|^{2} \bar{B} \\
& +t(1-t)^{2}\left\|z-x_{2}\right\|^{2} \bar{B}+t(1-t)\|u-v\|^{2} \bar{B} .
\end{aligned}
$$

Using this equality and applying three times condition (2) in the definition of strong $t$-convexity, we obtain

$$
\begin{aligned}
& t(1-t) F\left(x_{1}\right)+t(1-t) F\left(x_{2}\right)+(1-2 t(1-t)) F(z)+\frac{c}{2} t(1-t)\left\|x_{1}-x_{2}\right\|^{2} \bar{B} \\
& \subset t\left[(1-t) F\left(x_{1}\right)+t F(z)+c t(1-t)\left\|x_{1}-z\right\|^{2} \bar{B}\right] \\
& \quad+(1-t)\left[(1-t) F(z)+t F\left(x_{2}\right)+c t(1-t)\left\|z-x_{2}\right\|^{2} \bar{B}\right]+c t(1-t)\|u-v\|^{2} \bar{B} \\
& \quad \subset t F(u)+(1-t) F(v)+c t(1-t)\|u-v\|^{2} \bar{B} \\
& \quad \subset F(z) \subset 2 t(1-t) F(z)+(1-2 t(1-t)) F(z) .
\end{aligned}
$$

Since the set $F(z)$ is bounded closed and (by Lemma 2) convex, using the Rådström cancelation law, we get

$$
t(1-t) F\left(x_{1}\right)+t(1-t) F\left(x_{2}\right)+\frac{c}{2} t(1-t)\left\|x_{1}-x_{2}\right\|^{2} \bar{B} \subset 2 t(1-t) F(z) .
$$

Hence, dividing by $2 t(1-t)$, we obtain

$$
\frac{1}{2} F\left(x_{1}\right)+\frac{1}{2} F\left(x_{2}\right)+\frac{c}{4}\left\|x_{1}-x_{2}\right\|^{2} \bar{B} \subset F\left(\frac{x_{1}+x_{2}}{2}\right),
$$

which proves that $F$ is strongly midconvex with modulus $c$.

Remark 11 From Theorem 10 and Theorem 4 we infer that if a set-valued map $F: D \rightarrow$ $\operatorname{bcl}(Y)$ is strongly $t$-convex with modulus $c$ and upper semicontinuous, then it is strongly convex with modulus $c$. Similarly we can reformulate Corollaries 5, 6 and 7 for strongly $t$-convex set-valued maps. 


\section{A characterization of inner product spaces}

In this section we give some relationships between convex and strongly convex set-valued maps in the case where $X$ is a real inner product space (that is, the norm $\|\cdot\|$ is induced by an inner product: $\|x\|^{2}:=<x \mid x>$ ). Namely, we prove that a set-valued map $F$ is strongly convex with modulus $c$ if and only if $G=F+c\|\cdot\|^{2} \bar{B}$ is convex. Next, using this condition, we obtain a new characterization of inner product spaces among normed spaces.

Theorem 12 Let $(X,\|\cdot\|)$ be a real inner product space, $D$ be a convex subset of $X$ and $t \in(0,1)$ be a fixed number. A set-valued map $F: D \rightarrow \operatorname{ccl}(Y)$ is strongly $t$-convex with modulus $c$ if and only if the set-valued map $G$ defined by $G(x)=F(x)+c\|x\|^{2} \bar{B}, x \in D$, is $t$-convex.

Proof Assume first that $G$ is $t$-convex, that is

$$
t G\left(x_{1}\right)+(1-t) G\left(x_{2}\right) \subset G\left(t x_{1}+(1-t) x_{2}\right), \quad x_{1}, x_{2} \in D .
$$

Then

$$
\begin{gathered}
t F\left(x_{1}\right)+c t\left\|x_{1}\right\|^{2} \bar{B}+(1-t) F\left(x_{2}\right)+c(1-t)\left\|x_{2}\right\|^{2} \bar{B} \\
\subset F\left(t x_{1}+(1-t) x_{2}\right)+c\left\|t x_{1}+(1-t) x_{2}\right\|^{2} \bar{B} .
\end{gathered}
$$

By elementary properties of the inner product we get

$$
t\left\|x_{1}\right\|^{2}+(1-t)\left\|x_{2}\right\|^{2}=t(1-t)\left\|x_{1}-x_{2}\right\|^{2}+\left\|t x_{1}+(1-t) x_{2}\right\|^{2} .
$$

Therefore, (10) can be rewritten in the form

$$
\begin{aligned}
& t F\left(x_{1}\right)+(1-t) F\left(x_{2}\right)+c t(1-t)\left\|x_{1}-x_{2}\right\|^{2} \bar{B}+c\left\|t x_{1}+(1-t) x_{2}\right\|^{2} \bar{B} \\
& \quad \subset F\left(t x_{1}+(1-t) x_{2}\right)+c\left\|t x_{1}+(1-t) x_{2}\right\|^{2} \bar{B} .
\end{aligned}
$$

Since the set $F\left(t x_{1}+(1-t) x_{2}\right)$ is convex closed and $\bar{B}$ is bounded, by the Rådström cancelation law we obtain

$$
t F\left(x_{1}\right)+(1-t) F\left(x_{2}\right)+c t(1-t)\left\|x_{1}-x_{2}\right\|^{2} \bar{B} \subset F\left(t x_{1}+(1-t) x_{2}\right),
$$

which proves that $F$ is strongly $t$-convex with modulus $c$.

Conversely, if $F$ is strongly $t$-convex with modulus $c$, then

$$
\begin{aligned}
& t G\left(x_{1}\right)+(1-t) G\left(x_{2}\right) \\
& \quad=t F\left(x_{1}\right)+(1-t) F\left(x_{2}\right)+c t\left\|x_{1}\right\|^{2} \bar{B}+c(1-t)\left\|x_{2}\right\|^{2} \bar{B} \\
& \quad=t F\left(x_{1}\right)+(1-t) F\left(x_{2}\right)+c t(1-t)\left\|x_{1}-x_{2}\right\|^{2} \bar{B}+c\left\|t x_{1}+(1-t) x_{2}\right\|^{2} \bar{B} \\
& \quad \subset F\left(t x_{1}+(1-t) x_{2}\right)+c\left\|t x_{1}+(1-t) x_{2}\right\|^{2} \bar{B}=G\left(t x_{1}+(1-t) x_{2}\right),
\end{aligned}
$$

which shows that $G$ is $t$-convex.

The assumption that $X$ is an inner product space is not redundant in the above theorem. Moreover, the fact that $F$ is strongly $t$-convex with modulus $c$ if and only if $G=F+c\|\cdot\|^{2} \bar{B}$ is $t$-convex, characterizes inner product spaces among all normed spaces. The next theorem is motivated by [24], where similar characterizations of inner product spaces by strongly convex and strongly midconvex functions are presented.

Theorem 13 Let $(X,\|\cdot\|)$ be a real normed space. The following conditions are equivalent:

1. $(X,\|\cdot\|)$ is an inner product space; 
2. For everyc $>0$ and $t \in(0,1)$ and for every set-valued map $F: D \rightarrow \operatorname{ccl}(Y)$ defined on a convex subset $D$ of $X, F$ is stronglyt-convex with modulus $c$ if and only if $G=F+c\|\cdot\|^{2} \bar{B}$ is $t$-convex;

3. For every $c>0$ and for every set-valued map $F: D \rightarrow \operatorname{ccl}(Y)$ defined on a convex subset $D$ of $X$, if $G=F+c\|\cdot\|^{2} \bar{B}$ is midconvex then $F$ is strongly midconvex with modulus $c$.

Proof Implication $1 \Rightarrow 2$ follows by Theorem 10 . Implication $2 \Rightarrow 3$ is obvious.

To show that $3 \Rightarrow 1$, fix $x_{1}, x_{2} \in X$, take $r \geq \max \left\{\left\|x_{1}\right\|,\left\|x_{2}\right\|\right\}$ and consider the setvalued map $F=c\left(r^{2}-\|\cdot\|^{2}\right) \bar{B}$ defined on $D=\{x \in X:\|x\| \leq r\}$. By the assumption, $F$ is strongly midconvex with modulus $c$ because $G=F+c\|\cdot\|^{2} \bar{B}=c r^{2} \bar{B}$ is midconvex. Thus

$$
\frac{1}{2} F\left(x_{1}\right)+\frac{1}{2} F\left(x_{2}\right)+\frac{c}{4}\left\|x_{1}-x_{2}\right\|^{2} \bar{B} \subset F\left(\frac{x_{1}+x_{2}}{2}\right),
$$

whence, by the definition of $F$,

$$
\frac{c}{2}\left(r^{2}-\left\|x_{1}\right\|^{2}\right) \bar{B}+\frac{c}{2}\left(r^{2}-\left\|x_{2}\right\|^{2}\right) \bar{B}+\frac{c}{4}\left\|x_{1}-x_{2}\right\|^{2} \bar{B} \subset c\left(r^{2}-\left\|\frac{x_{1}+x_{2}}{2}\right\|^{2}\right) \bar{B} .
$$

From here

$$
\left(4 r^{2}-2\left\|x_{1}\right\|^{2}-2\left\|x_{2}\right\|^{2}+\left\|x_{1}-x_{2}\right\|^{2}\right) \bar{B} \subset\left(4 r^{2}-\left\|x_{1}+x_{2}\right\|^{2}\right) \bar{B},
$$

and, consequently,

$$
\left\|x_{1}+x_{2}\right\|^{2}+\left\|x_{1}-x_{2}\right\|^{2} \leq 2\left\|x_{1}\right\|^{2}+2\left\|x_{2}\right\|^{2} .
$$

This inequality holds for all $x_{1}, x_{2} \in X$. Putting $u=x_{1}+x_{2}$ and $v=x_{1}-x_{2}$ in (11) we get also

$$
2\|u\|^{2}+2\|v\|^{2} \leq\|u+v\|^{2}+\|u-v\|^{2}, \quad u, v \in X .
$$

Conditions (11) and (12) mean that the norm $\|\cdot\|$ satisfies the parallelogram law, which implies, by the classical Jordan-von Neumann theorem, that $(X,\|\cdot\|)$ is an inner product space.

\section{Relationships with strongly convex sets}

Let $(Z,\|\cdot\|)$ be a real normed space and $R>0$. Given $z_{1}, z_{2} \in Z$ with $\left\|z_{1}-z_{2}\right\| \leq 2 R$, we denote by $D_{R}\left(z_{1}, z_{2}\right)$ the intersection of all closed balls with radius $R$ containing $z_{1}$ and $z_{2}$. A set $A \subset Z$ is called strongly convex with respect to the radius $R$ if $\operatorname{diam} A \leq 2 R$ and $D_{R}\left(z_{1}, z_{2}\right) \subset A$ for all $z_{1}, z_{2} \in A$. Equivalently, $A$ is strongly convex with respect to $R$ if it can be represented as the intersection of a family of closed balls of radius $R$ (see $[26,35,36])$. Strongly convex sets appear naturally during the study of accessible sets in nonlinear control systems, and they were considered, among others, by Pliś [25], Łojasiewicz [18], Frankowska and Olech [10] (they called these sets $R$-regular or $R$-convex sets). Many properties and applications of strongly convex sets can be found in Polovinkin [26] and Vial $[35,36]$. In this section we present some relations between strongly convex set-valued maps and strongly convex sets.

In [26] Polovinkin proved the following theorem: If $A \subset \mathbb{R}^{n} \times \mathbb{R}$ is a strongly convex set with respect to a radius $R$ and $D=\left\{x \in \mathbb{R}^{n}: \exists \alpha \in \mathbb{R},(x, \alpha) \in A\right\}$, then the function 
$f: D \rightarrow \mathbb{R}$ defined by $f(x)=\inf \{\alpha \in \mathbb{R}:(x, \alpha) \in A\}$ is strongly convex with modulus $c=\frac{1}{2 R}$. Using this result we can show that every strongly convex set $A \subset \mathbb{R}^{n} \times \mathbb{R}$ is the graph of a strongly convex set-valued map. Recall that the graph of $F: D \rightarrow n(Y)$ is defined by $\operatorname{Gr} F=\{(x, y): x \in D, y \in F(x)\}$.

Theorem 14 Let $A \subset \mathbb{R}^{n} \times \mathbb{R}$ be a strongly convex set with respect to a radius $R>0$ and let $D=\left\{x \in \mathbb{R}^{n}: \exists \alpha \in \mathbb{R},(x, \alpha) \in A\right\}$. Then there exists a set-valued map $F: D \rightarrow b c l(\mathbb{R})$ strongly convex with modulus $\frac{1}{2 R}$ such that $\operatorname{Gr} F=A$.

Proof Denote $f_{1}(x)=\min \{\alpha \in \mathbb{R}:(x, \alpha) \in A\}$ and $f_{2}(x)=\max \{\alpha \in \mathbb{R}:(x, \alpha) \in A\}$, $x \in D$. By the theorem of Polovinkin mentioned above, $f_{1}$ is strongly convex with modulus $\frac{1}{2 R}$ and $f_{2}$ is strongly concave with modulus $\frac{1}{2 R}$. Hence the set-valued map $F: D \rightarrow b c l(\mathbb{R})$ defined by $F(x)=\left[f_{1}(x), f_{2}(x)\right]$ is also strongly convex with modulus $\frac{1}{2 R}$ (cf. Example 1 ) and $G r F=A$.

The following example shows that the converse theorem is not true.

Example 2 Consider the set-valued map $F:[-R, R] \rightarrow c(\mathbb{R})$ defined by $F(x)=$ $\left[-\sqrt{R^{2}-x^{2}}, 1+\sqrt{R^{2}-x^{2}}\right], \quad x \in[-R, R]$. By the known characterization of twice differentiable strongly convex functions stating that $f$ is strongly convex with modulus $c$ if and only if $f^{\prime \prime} \geq 2 c$, we infer that $f_{1}(x)=-\sqrt{R^{2}-x^{2}}$ is strongly convex with modulus $\frac{1}{2 R}$. Similarly, $f_{2}(x)=1+\sqrt{R^{2}-x^{2}}$ is strongly concave with modulus $\frac{1}{2 R}$. Hence $F$ is strongly convex with modulus $\frac{1}{2 R}$. However, the graph of $F$ is not strongly convex with respect to the radius $R$ because diam $G r F>2 R$. In fact, $G r F$ is not strongly convex with respect to any radius $r>0$. Indeed, for $2 r<\operatorname{diam} G r F$ it is clear, and for $2 r \geq \operatorname{diam} G r F$ it follows from the fact that $(R, 0),(R, 1) \in G r F$ but $D_{r}((R, 0),(R, 1)) \nsubseteq G r F$.

In [35] Vial proved the following theorem: A closed set $A \subset \mathbb{R}^{n}$ is strongly convex with respect to a radius $R$ if and only if $A$ is bounded and for any $z_{1}, z_{2} \in A$ and $t \in(0,1)$ the closed ball $K\left(z_{t}, \delta_{t}\right)$ with center $z_{t}=t z_{1}+(1-t) z_{2}$ and radius $\delta_{t}=\frac{1}{2 R} t(1-t)\left\|z_{1}-z_{2}\right\|^{2}$, is included in $A$. In the same way one can prove that this result holds also for every set $A \subset X \times Y$, where $X$ and $Y$ are arbitrary real inner product spaces. Then $X \times Y$ is also an inner product space with the inner product $<\left(x_{1}, y_{1}\right)\left|\left(x_{2}, y_{2}\right)>=<x_{1}\right| x_{2}>+<y_{1}\left|y_{2}\right\rangle$ and the norm $\|(x, y)\|^{2}=\|x\|^{2}+\|y\|^{2}$.

Using the above result we can obtain the following generalization of Theorem 14.

Theorem 15 Let $X, Y$ be real inner product spaces, $A \subset X \times Y$ be a strongly convex set with respect to a radius $R>0$ and let $D=\{x \in X: \exists y \in \mathbb{R},(x, y) \in A\}$. Then there exists a set-valued map $F: D \rightarrow \operatorname{bcl}(Y)$ strongly convex with modulus $\frac{1}{2 R}$ such that $G r F=A$.

Proof Consider the set-valued map $F: D \rightarrow \operatorname{bcl}(Y)$ defined by

$$
F(x)=A_{x}:=\{y \in Y:(x, y) \in A\} .
$$

Clearly, $\operatorname{Gr} F=A$. We will show that $F$ is strongly convex with modulus $\frac{1}{2 R}$. To this aim fix $x_{1}, x_{2} \in D, y_{1} \in F\left(x_{1}\right), y_{2} \in F\left(x_{2}\right)$ and $t \in(0,1)$. By the Vial-type result mentioned above we have

$$
K\left(\left(x_{t}, y_{t}\right), \delta_{t}\right) \subset A,
$$

where $K\left(\left(x_{t}, y_{t}\right), \delta_{t}\right)$ is the closed ball in $X \times Y$ with center $\left(x_{t}, y_{t}\right)=t\left(x_{1}, y_{1}\right)+(1-t)$ $\left(x_{2}, y_{2}\right)$ an radius $\delta_{t}=\frac{1}{2 R} t(1-t)\left\|\left(x_{1}, y_{1}\right)-\left(x_{2}, y_{2}\right)\right\|^{2}$. Taking the $x_{t}$-section on the both sides we get

$$
K\left(\left(x_{t}, y_{t}\right), \delta_{t}\right)_{x_{t}} \subset A_{x_{t}},
$$


and from here

$$
t y_{1}+(1-t) y_{2}+\frac{1}{2 R} t(1-t)\left\|x_{1}-x_{2}\right\|^{2} \bar{B} \subset F\left(t x_{1}+(1-t) x_{2}\right) .
$$

Since this inclusion holds for all $y_{1} \in F\left(x_{1}\right)$ and $y_{2} \in F\left(x_{2}\right)$, we have also

$$
t F\left(x_{1}\right)+(1-t) F\left(x_{2}\right)+\frac{1}{2 R} t(1-t)\left\|x_{1}-x_{2}\right\|^{2} \bar{B} \subset F\left(t x_{1}+(1-t) x_{2}\right),
$$

which shows that $F$ is strongly convex with modulus $\frac{1}{2 R}$ and finishes the proof.

Acknowledgments The authors are grateful to the Referees and the Editors for helpful comments and suggestions.

Open Access This article is distributed under the terms of the Creative Commons Attribution License which permits any use, distribution, and reproduction in any medium, provided the original author(s) and the source are credited.

\section{References}

1. Aleman, A.: On some generalization of convex sets and convex functions. Anal. Numér. Théor. Approx. 14, 1-6 (1985)

2. Azócar, A., Giménez, J., Nikodem, K., Sánchez, J.L.: On strongly midconvex functions. Opuscula Math. 31, 15-26 (2011)

3. Benoist, J., Popovici, N.: Generalized convex set-valued maps. J. Math. Anal. Appl. 288, 161-166 (2003)

4. Borwein, J.M.: Multivalued convexity and optimization: a unified approach to inequality and equality constrains. Math. Program. 13, 183-199 (1977)

5. Borwein, J.M.: Convex relations in analysis and optimization. In: Schaible, S., Ziemba, W. (eds.) Generalized Convexity in Optimization and Economics, pp. 335-377. Academic Press, New York (1981)

6. Breckner, W.W.: Continuity of generalized convex and generalized concave set-valued functions. Rev. Anal. Numér. Théor. Approx. 22, 39-51 (1993)

7. Cardinali, T., Nikodem, K., Papalini, F.: Some results on stability and on characterization of $K$-convexity of set-valued functions. Ann. Polon. Math. 58, 185-192 (1993)

8. Daróczy, Z., Zs, Páles: Convexity with given infinite weight sequences. Stochastica 11, 5-12 (1987)

9. Enkhbat, R., Barsbold, B., Kamada, M.: A numerical approach for solving some convex maximization problems. J. Glob. Optim. 35, 85-101 (2006)

10. Frankowska, H., Olech, C.: $R$-convexity of the integral of the set-valued functions. In: Clark, D.N., Pecelli, G., Sacksteder, R. (eds.) Contributions to Analysis and Geometry, pp. 117-129. Johns Hopkins University Press, Baltimore (1981)

11. Huang, H.: Global error bounds with exponents for multifunctions with set constraints. Commun. Contemp. Math. 12, 417-435 (2010)

12. Huang, H.: Inversion theorem for nonconvex multifunctions. Math. Inequal. Appl. 13, 841-849 (2010)

13. Jeyakumar, V.: On subgradient duality with strong and weak convex functions. J. Aust. Math. Soc. 40, 143-152 (1986)

14. Karamardian, S.: The nonlinear complementarity problem with applications, Part 2. J. Optim. Theory Appl. 4, 167-181 (1969)

15. Kuczma, M.: An Introduction to the Theory of Functional Equations and Inequalities. Cauchy's Equation and Jensen's Inequality, PWN-Uniwersytet Sląski, Warszawa-Kraków-Katowice, 1985, 2nd edn. Birkhäuser, Basel (2009)

16. Kuhn, N.: A note on t-convex functions. In: Walter, W. (ed.) General Inequalities, 4 (Oberwolfach, 1983), International Series of Numerical Mathematics, vol. 71, pp. 269-276. Birkhäuser, Basel (1984)

17. Langenberg, N., Tichatschke, R.: Interior proximal methods for quasiconvex optimization. J. Glob. Optim. 52, 641-661 (2012)

18. Łojasiewicz Jr, S.: Some properties of accessible sets in non linear control systems. Ann. Polon. Math 37, 123-127 (1979)

19. Lin, G.H., Fukushima, M.: Some exact penalty results for nonlinear programs and mathematical programs with equilibrium constraints. J. Optim. Theory Appl. 118, 67-80 (2003) 
20. Merentes, N., Nikodem, K.: Remarks on strongly convex functions. Aequationes Math. 80, 193-199 (2010)

21. Nikodem, K.: On midpoint convex set-valued functions. Aequationes Math. 3, 46-56 (1987)

22. Nikodem, K.: $K$-Convex and $K$-Concave Set-Valued Functions, Zeszyty Nauk. Politech. Łódz. Mat. 559 (Rozprawy Nauk 114), Łódź, pp. 1-75 (1989)

23. Nikodem, K.: Continuity properties of convex-type set-valued maps. J. Inequal. Pure Appl. Math. 4(3), Art. 52 (2003)

24. Nikodem, K., Zs, Páles: Characterizations of inner product spaces by strongly convex functions. Banach J. Math. Anal. 5(1), 83-87 (2011)

25. Pliś, A.: Accessible sets in control theory. In: International Conference on Differential Equations (Univ. of Southern California), pp. 646-650. Academic Press, New York (1975)

26. Polovinkin, E.: Strongly convex analysis. Sbornik Math. 187, 259-286 (1996)

27. Polyak, B.T.: Existence theorems and convergence of minimizing sequences in extremum problems with restrictions. Sov. Math. Dok1. 7, 72-75 (1966)

28. Rådström, H.: An embedding theorem for space of convex sets. Proc. Am. Math. Soc. 3, 165-169 (1952)

29. Rajba, T., Wąsowicz, Sz.: Probabilistic characterization of strong convexity. Opuscula Math. 31, 97-103 (2011)

30. Ricceri, B.: Remarks on multifunctions with convex graph. Arch. Math. 52, 519-520 (1989)

31. Roberts, A.W., Varberg, D.E.: Convex Functions. Academic Press, New York (1973)

32. Robinson, S.M.: Regularity and stability for convex multivalued functions. Math. Oper. Res. 1, 130-143 (1976)

33. Sterna-Karwat, A.: Convexity of the optimal multifunctions and its consequences in vector optimization. Optimization 20, 799-808 (1989)

34. Thibault, L.: Continuity of measurable convex multifunctions. In: Multifunctions and Integrands. Lecture Notes in Math, pp. 216-224. Springer, Berlin (1091)

35. Vial, J.P.: Strong convexity of sets and functions. J. Math. Econ. 9, 187-205 (1982)

36. Vial, J.P.: Strong and weak convexity of sets and functions. Math. Oper. Res. 8, 231-259 (1983)

37. Zavriev, S.K.: On the global optimization properties of finite difference local descent algorithms. J. Glob. Optim. 3, 67-78 (1993) 